\title{
Fibrosis regression after treatment with Daas
}

\begin{abstract}
Background: HCV infection promotes liver fibrogenesis by direct and indirect mechanisms through chronic inflammation. Successful HCV treatment was associated with significant improvement in liver histology. Non-invasive methods (like APRI score and Fib-4 index) for the evaluation of liver fibrosis have been developed and validated.
\end{abstract}

Methods: Fib-4 index and APRI score were calculated for 50 Egyptian chronic HCV (genotype 4) patients who received sofosbuvir (400 mg once daily) plus simeprevir (150 $\mathrm{mg}$ once daily) for 12 weeks. The end-point was a sustained virological response at 12 (SVR12) weeks post treatment.

Results: Baseline fibrosis stage showed a mean APRI score of 0.63188 and mean Fib-4 index of 1.97210. All patients had an end of treatment response with negative HCV RNA. Improvement in liver enzymes and platelet count was reflected on the regression of fibrosis after treatment with a significant reduction in levels of both APRI score and Fib-4 index.

Conclusion: Fibrosis regression -assessed by non-invasive markers of fibrosis - is achievable upon removal of the causative agent.

\author{
Volume 7 Issue I - 2017 \\ Mai Ismail Mehrez,' Mohamed A Saleh, ${ }^{2}$ Azza \\ Fareid' \\ 'Fellow of Hepatology, National Hepatology and Tropical \\ Medicine Research Institute, Egypt \\ ${ }^{2}$ Fellow of Internal Medicine, National Hepatology and Tropical \\ Medicine Research Institute, Egypt
}

Correspondence: Mai Ismail Mehrez, Fellow of Hepatology, National Hepatology and Tropical Medicine Research Institute, Egypt, Email maymehrezmd@yahoo.com

Received: May 09, 2017| Published: June 19, 2017

\section{Introduction}

Hepatitis $\mathrm{C}$ is a worldwide problem with a prevalence estimated to be $3 \%$ according to the World Health Organization (WHO). Egypt has the highest prevalence of $\mathrm{HCV}$ which was estimated to be $7.3 \%$ with predominance of genotype $4 .{ }^{1}$ Acute $\mathrm{HCV}$ infection usually lead to no symptoms and about $75 \%$ of cases infected with $\mathrm{HCV}$ reach a chronic stage leading to chronic $\mathrm{HCV}$ infection. ${ }^{2} \mathrm{HCV}$ infection promote liver fibrogenesis by direct and indirect mechanisms through chronic inflammation. ${ }^{3}$ Sequelae of chronic HCV may lead to liver cirrhosis in nearly $20-50 \%$ of patients infected, this may be complicated by liver cell failure and hepatocellular carcinoma later on.

Successful HCV treatment was associated with significant improvement in liver histology in patients who underwent follow up liver biopsy after treatment. ${ }^{4}$ However, there are drawbacks associated with liver biopsy, such as its invasiveness, cost, inter- and intraobserver variability, and sampling error, all of which serve to limit its usefulness. For this reason, non-invasive methods for the evaluation of liver fibrosis have been developed and validated. ${ }^{5}$

A broad range of noninvasive markers is under intensive study to complement or replace biopsy in future trials. These include the APRI score and Fib-4 index. .The AST/platelet ratio (APRI) was developed by Wai and his group. It is measured as (AST/upper limit of normal $x$ 100)/platelet count. In the original study, A cutoff of $>1.5$ was considered correlating with advanced fibrosis F3-F4 and cirrhosis. ${ }^{6}$ APRI was considered of high accuracy also in predicting advanced fibrosis in different forms of liver disease. ${ }^{7}$ The FIB- 4 score is a score calculated based on age, platelet count, AST, and ALT according to the following formula

Age $[\mathrm{yrs}] \times \mathrm{AST} /$ platelet count $\left[\mathrm{Plt} \times 10^{9} / \mathrm{L}\right] \times(\mathrm{ALT} 1 / 2[\mathrm{U} / \mathrm{L}])$.

It was first developed by Sterling et al. ${ }^{8}$ to assess fibrosis in HIV/ HCV coinfected patients with a cutoff value of $3.25 .{ }^{8}$ The Fib-4 score was then validated for detection of the monoinfection HCV. ${ }^{9}$ The aim of our study was to assess the possible fibrosis regression in patients chronically infected with HCV through the indirect markers APRI and Fib-4 index.

\section{Patients and methods}

Fifty Egyptian patients chronically infected with HCV genotype 4 were enrolled; they received sofosbuvir plus simeprevir in the National Hepatology and Tropical Medicine research Institute (NHTMRI) in Cairo, Egypt. The work was carried out in accordance with Helsinki declaration, and following the guidelines of the ethical approval committees in Egypt. Patients were included according to the approved treatment recommendations by EASL 2015. ${ }^{10}$

All patients had HCV RNA positivity, their age ranged between 18-70, they were either naïve to HCV treatment or had a previous treatment experience whether interferon based or sofosbuvir based and no restrictions were put on either BMI or fibrosis stage. Patients who were excluded were those who had hypersensitivity to the drugs used, pregnants or breast feeding, poorly controlled diabetics (HbA1C $>8$ ), patients with decompensated liver disease such as those with Ascites, history of Ascites, history of hepatic encephalopathy and hepatocellular carcinoma (except 4 weeks after intervention aiming at cure with no evidence of activity by dynamic imaging). Also, patients with renal disease in the form of serum creatinine $>2.5 \mathrm{mg} /$ $\mathrm{dl}$ or eGFR $<30 \mathrm{ml} / \mathrm{min}$. as well as patients with INR $>1.7$, serum albumin $<2.8 \mathrm{~g} / \mathrm{dl}$, total bilirubin $>3 \mathrm{mg} / \mathrm{dl}$ or platelet count $<50,000 /$ $\mathrm{mm}^{3}$ were excluded. ${ }^{11}$

All patients were subjected to full history taking, including history of other causes of chronic liver disease. They were investigated using the following laboratory investigations: Liver biochemical profile including total and direct Bilirubin, Aspartate transaminase (AST), Alanine transaminase (ALT), Albumin (ALB), International normalized ratio (INR). Also complete blood count (CBC), HBsAg, HCV PCR quantitative, serum Creatinine, Alpha feto protein (AFP), and Fasting blood sugar (FBS), HbAlc if diabetic. Pregnancy test was done for female patients in childbearing period. Fib-4 index was calculated to all patients using the formula: age (years) X AST [U/L]/ (platelets $\left[10^{9} / \mathrm{L}\right]$ X $(\text { ALT }[\mathrm{U} / \mathrm{L}])^{1 / 2.8}$ APRI score was calculated too using this equation: AST/upper limit of normal x 100)/platelet count. ${ }^{6}$

Abdominal ultrasonography was done to each patient to detect echopattern of the liver (ultrasonographic features of cirrhosis), 
presence of signs of portal hypertension, and to exclude hepatocellular carcinoma. Patients were then categorized into easy and difficultto-treat groups to guide treatment plan nationwide, where easy to treat group included those who were noncirrhotic (by clinical \& ultrasonographic examination), Fib- $4<3.25$, albumin $>3.5$, total serum Bilirubin $<1.2 \mathrm{mg} / \mathrm{dL}, \mathrm{INR}<1.2$ and Platelet count $>=150000 \mathrm{~mm}^{3}$, while difficult-to-treat group included those who were cirrhotic (by clinical \& ultrasonographic examination), Fib-4 $>3,25$, albumin $\leq 3.5$, total serum Bilirubin $>1.2 \mathrm{mg} / \mathrm{dL}, \mathrm{INR}>1.2$, and platelet count $<150$ $000 \mathrm{~mm}^{3}{ }^{11}$

\section{Treatment regimen}

All patients received sofosbuvir $400 \mathrm{mg}$ once daily plus simeprevir $150 \mathrm{mg}$ once daily for 12 weeks. The end point was a sustained virological response at 12 (SVR12) weeks post treatment. The efficacy and toxicity of concurrent drugs given for comorbidities and potential drug-drug interactions were revised prior to initiation of therapy and whenever possible, an interacting co-medication stopped for the duration of HCV treatment or switched to an alternative drug with less interaction potentials.

\section{Monitoring of treatment efficacy}

Quantitative HCV-PCR was measured using the COBAS Ampliprep/COBAS TaqMan (CAP/CTM) assay. The CAP/CTM $\mathrm{HCV}$ test, v2.0 was performed according to the manufacturer's instructions. The assay was standardized to the World Health Organization international standard (WHO-IS) for HCV RNA (2nd WHO standard NIBSC code 96/798), and results were reported in international units per milliliter. HCV RNA was extracted from $650 \mu \mathrm{l}$ according to the manufacturer's instructions. The Cobas TaqMan 96 analyzer was used for automated real-time PCR amplification and detection of PCR products. The dynamic range of quantification was 15 to $100,000,000 \mathrm{IU} / \mathrm{ml}\left(1.2\right.$ to $\left.8.0 \log _{10} \mathrm{IU} / \mathrm{ml}\right)$, with the claimed lower limit of detection equal to the lower limit of quantification (15 $\mathrm{IU} / \mathrm{ml}$, i.e., $\left.1.2 \log _{10} \mathrm{IU} / \mathrm{ml}\right)$.

HCV quantitation was performed prior to treatment, and 12 weeks after treatment where virologic response was considered when HCV RNA is less than lower limit of detection week 12 post treatment (SVR12) while treatment failure was defined as confirmed HCV RNA above LLOQ 12 weeks post treatment. Treatment discontinuation due to adverse events was considered treatment failure. Statistical analysis was performed using SPSS version 21 for Windows (IBM Corp., Armonk, NY). Continuous variables were presented by means and standard deviations (SD) and were analyzed using student's unpaired t-test. Mann Whitney Willcoxon U test was used instead of unpaired t-test in non-parametric data (SD $>50 \%$ mean). Categorical variables by frequencies and percentages and were analyzed using Chi-square whenever appropriate.

\section{Results}

This study included 50 Egyptian patients with HCV Genotype 4 infection. Twenty-seven patients (54\%) were males, mean age was 52.92 years, liver cirrhosis was detected in $21(42 \%)$ patients and only four patients $(8 \%)$ had previous treatment to $\mathrm{HCV}$, whether interferon or sofosbuvir based. Only one patient had a history of previous hepatocellular carcinoma (HCC), which was well ablated as documented by dynamic imaging 4 weeks before antiviral therapy. Regarding other comorbidities, 11 patients $(22 \%)$ were hypertensives and 7 patients (14\%) were diabetics (Table 1).

Baseline laboratory values did not show any abnormalities except the increase in AST, ALT and AFP $(46.17 \pm 22.072 \mathrm{IU} / \mathrm{ml}$, $51.22 \pm 25.237 \mathrm{IU} / \mathrm{ml}, 8.75 \pm 12.328 \mathrm{ng} / \mathrm{ml}$ respectively). Baseline HCV RNA by PCR was $1707482.54 \pm 5377401.880 \mathrm{IU} / \mathrm{ml}$. Baseline fibrosis stage showed a mean APRI score of 0.63188 and mean Fib-4 index of 1.97210 (Table 2). All patients had an end of treatment response with negative HCV RNA while $5(10 \%)$ patients suffered recurrence of infection after stopping antiviral therapy and 45 patients (90\%) had negative HCV RNA at 12 weeks post treatment (SVR12) (Table 3). Regarding liver functions, a significant improvement occurred at the levels of AST, Albumin, Bilirubin, Platelets and INR while a nonsignificant improvement occurred in ALT level. Moreover, AFP level significantly reduced after treatment. Improvement in liver enzymes and platelet count was reflected on the regression of fibrosis after treatment with a significant reduction in levels of both APRI score and Fib-4 index (Table 4).

Table I Demographics and patients characteristics

\begin{tabular}{ll}
\hline Mean (STD) & \\
\hline Age & $52.92(\mathrm{I} 3.4 \mathrm{I} 3)$ \\
\hline & Number (\%) \\
\hline Male/Female & $27(54 \%) / 23(46 \%)$ \\
Cirrhotics & $2 \mathrm{I}(42 \%)$ \\
DM & $7(\mathrm{I} \%)$ \\
HTN & $\mathrm{I} \mathrm{I}(22 \%)$ \\
Previous treatment experience & $4(8 \%)$ \\
HCC & $\mathrm{I}(2 \%)$ \\
\hline
\end{tabular}

Table 2 Baseline laboratory values and fibrosis scores

\begin{tabular}{ll}
\hline Mean (STD) & \\
\hline Bilirubin & $0.76(0.3 \mid 4)$ \\
AST & $46.17(22.072)$ \\
ALT & $51.22(25.237)$ \\
Albumin & $4.05(0.439)$ \\
INR & $1.07(0.085)$ \\
Creatinine & $0.82(0.202)$ \\
Hb & $I 4.02(1.458)$ \\
WBCs & $6.69(\mathrm{I} .844)$ \\
PLTs & $212.60(65.674)$ \\
AFP & $8.75(\mathrm{I} 2.328)$ \\
HCV RNA & $\mathrm{I} 707482.54(537740 \mathrm{I} .880)$ \\
APRI & $0.63188(0.450768)$ \\
Fib-4 & $\mathrm{I} .97210(\mathrm{I} .383455)$ \\
\hline
\end{tabular}

Table 3 Treatment response

\begin{tabular}{ll}
\hline Number (\%) & \\
\hline End of treatment & $50(100 \%)$ \\
SVRI2 & $45(90 \%)$ \\
\hline
\end{tabular}


Table 4 Correlation between pretreatment and post-treatment parameters

\begin{tabular}{lll}
\hline & Correlation & Significance \\
\hline W0_Tbil \&W24_Tbil & 0.458 & 0.001 \\
W0_AST \&W24_AST & 0.46 & 0.001 \\
W0_ALT \&W24_ALT & 0.229 & 0.11 \\
W0_ALB \&W24_ALB & 0.419 & 0.002 \\
W0_INR \&W24_INR & 0.369 & 0.008 \\
W0_Creat \&W24_Creat & 0.456 & 0.001 \\
W0_HB \&W24_HB & 0.703 & 0 \\
W0_WBCs \&W24_WBCs & 0.539 & 0 \\
W0_PLT \&W24_PLT & 0.761 & 0 \\
W0_AFP \&W24_AFP & 0.814 & 0 \\
W0_PCRQN \&W24_PCRQN & -0.05 & 0.732 \\
W0_APRI \&W24_APRI & 0.599 & 0 \\
W0_FIB4 \&W24_FIB4 & 0.724 & 0 \\
\hline
\end{tabular}

\section{Discussion}

Eradicating the underlying liver disease is the key for prevention of fibrosis progression. For example, in case of $\mathrm{HCV}$, achievement of sustained virological response (SVR) is directly related to a reduction in the amount of scarring in the liver and hence resolution of fibrosis, although it might take several years for this to occur, the same way that it took several years for fibrosis to develop. Assessment of fibrosis in the liver can be done through studying liver function tests that assess fibrosis based on measuring other elements that correlate with fibrosis in the liver. In this study, we depended on the non-invasive biomarkers for fibrosis APRI and Fib-4 index which showed significant reduction of about $66 \%$ and $36.8 \%$ respectively compared to baseline values denoting fibrosis regression among patients who received antiviral treatment.

This study was a little bit superior to a study done by Bachofner and his colleagues who observed a reduction in APRI scores and Fib-4 index after $\mathrm{HCV}$ eradication by about $60.9 \%$ and $29.1 \%$ respectively. ${ }^{12}$ Another study done by Bruno and colleagues in 2016 compared APRI score and Fib-4 index before treatment and 24 months post treatment in 84 chronic HCV patients, all of whom achieved SVR24 and 77.3\% were cirrhotics. APRI score was shown to improve by $36.7 \%$ of its baseline value while Fib-4 index reduced by $67.2 \%$. These results were matching with results in our study despite including higher portion of cirrhotics and longer follow up period compared to our study. ${ }^{13}$

In 2015, a meta-analysis was done by Akhtar, where out of 443 patients, 137 achieved SVR and cirrhosis was found to regress in about $53 \%$ of the patients as documented by liver biopsy pretreatment and a follow up liver biopsy after treatment. This study was superior to results of our study; however, it was shown that most patients who had fibrosis regression were found to have their follow up liver biopsy after more than 36 months post treatment, unlike our study where APRI and Fib-4 were done 3 months only after treatment. ${ }^{14}$

\section{Conclusion}

This study shows that fibrosis regression is achievable upon removal of the causative agent. The development of non-invasive markers for assessment of fibrosis showed a great reliability, avoiding the need for using a liver biopsy with its major drawbacks and invasiveness.

\section{Acknowledgments}

None.

\section{Conflicts of interest}

Authors declare that there is no conflict of interest.

\section{References}

1. Waked I, Doss W, El-Sayed M, et al. The current and future disease burden of chronic hepatitis C virus infection in Egypt. Arab $J$ Gastroenterol. 2014;15(2):45-52.

2. Bonkovsky HL, Mehta S. Hepatitis C: a review and update. J Am Acad Dermatol. 2001;44(2):159-182.

3. S Petta. Insulin resistance and diabetes mellitus in patients with chronic hepatitis C: spectators or actors?" Digestive and Liver Disease. 2012;44(5):359-360.

4. Poynard T, McHutchison J, Manns M, et al. Impact of pegylated interferon alfa- $2 \mathrm{~b}$ and ribavirin on liver fibrosis in patients with chronic hepatitis C. Gastroenterology. 2002;122(2002):1303-1313.

5. Cadranel JF, Rufat P, Degos F. Practices of liver biopsy in France: results of a prospective nationwide survey. For the Group of Epidemiology of the French Association for the Study of the Liver (AFEF). Hepatology. 2000;32(3):477-481.

6. Wai CT, Greenson JK, Fontana RJ, et al. A simple noninvasive index can predict both significant fibrosis and cirrhosis in patients with chronic hepatitis C. Hepatology. 2003;38(2):518-526.

7. Loaeza-del-Castillo A, Paz-Pineda F, Oviedo-Cárdenas E, et al. AST to platelet ratio index (APRI) for the noninvasive evaluation of liver fibrosis. Ann Hepatol. 2008;7(4):350-357.

8. Sterling RK, Lissen E, Clumeck N, et al. Development of a simple noninvasive index to predict significant fibrosis in patients with HIV/ HCV coinfection. Hepatology. 2006;43(6):1317-1325.

9. Vallet-Pichard A, Mallet V, Nalpas B, et al. FIB-4: an inexpensive and accurate marker of fibrosis in $\mathrm{HCV}$ infection. comparison with liver biopsy and fibrotest. Hepatology. 2007;46(1):32-36.

10. European Association for the Study of the Liver. EASL Clinical Practice Guidelines: management of hepatitis $\mathrm{C}$ virus infection. $J$ Hepatol. 2015;60(2):392-420

11. Guerra J, Garenne M, Mohamed MK, et al. HCV burden of infection in Egypt: results from a nationwide survey. $J$ Viral Hepat. 2012;19(8):560-567.

12. Bachofner JA, Valli PV, Kröger A, et al. DAA treatment of chronic hepatitis $\mathrm{C}$ results in rapid regression of transient elastography and fibrosis markers FIB-4 and APRI. Liver Int. 2017;37(3):369-376.

13. G Bruno, R Dell'Acqua, E Milano, et al. Early regression of liver fibrosis in HCV infected patients with Or without HIV infection after treatment with DAAs. ICAR; 2016. 127 p.

14. Akhtar E, Manne V, Saab S. Cirrhosis regression in hepatitis C patients with sustained virological response after antiviral therapy: a metaanalysis. Liver Int. 2015;35(1):30-36. 